\title{
Preparation, Characterization and Application of Carbon Nanotubes Wrapped Nanoflake-like $\mathrm{SnS}_{2}$ Composite
}

\author{
Juan $\mathrm{Li}^{\mathrm{a}}$, Xingzhang Qin ${ }^{\mathrm{b}}$ and Zhanjun Yang ${ }^{\mathrm{c}, *}$ \\ College of Chemistry and Chemical Engineering, Yangzhou University; Jiangsu Key Laboratory of \\ Environmental Engineering and Monitoring, Yangzhou 225002, PR China \\ alijuan@yahoo.cn; ${ }^{b}$ xingzhang qin@126.com; ${ }^{c}$ zjyang@yzu.edu.cn
}

Keywords: Nanocomposite; Nanoflake-like $\mathrm{SnS}_{2}$; Carbon nanotubes; Electrochemistry

\begin{abstract}
A novel multiwall carbon nanotubes wrapped nanoflake-like $\mathrm{SnS}_{2}\left(\mathrm{MWCNTs}_{\mathrm{SnS}}\right)$ composite was designed by simply mixing carbon nanotubes with nanoflake-like $\mathrm{SnS}_{2}$. The fabricated MWCNTs-SnS 2 composite material was characterized using scan electron microscopy and transmission electron microscopy. The MWCNTs-SnS $\mathrm{S}_{2}$ was used to modify glassy carbon electrode to examine the electrocatalysis of hydrogen peroxide and oxygen. Moreover, direct electrochemistry of glucose oxides immobilized on $\mathrm{MWCNTs}^{-\mathrm{SnS}_{2}}$ modified electrode was investigated. This $\mathrm{MWCNTs}-\mathrm{SnS}_{2}$ composite provided a promising approach to fabricate electrochemical sensor.
\end{abstract}

\section{Introduction}

Nanomaterials have been extensively used in many research areas due to their unique physical and chemical properties [1]. Recently, semiconductor nanomaterials have grabbed increasing attentions in the electrochemical sensing fields due to excellent properties [2-7]. $\mathrm{SnS}_{2}$ is an n-type semiconductor with a bandgap of 2.18-2.44 eV [8,9], and has good stability in acid and neutral aqueous solutions as well as certain oxidative and thermal stability in air, which makes it become a promising visible light-sensitive photocatalyst [10]. In addition, because of its intriguing electrical, optical and gas sensing properties, $\mathrm{SnS}_{2}$ has been chosen as a candidate material in solar cells and opto-electronic devices, electrode for lithium-ion batteries, pigment and gas sensor [11-13].

Composite nanomaterials that consist of nanocarbons in combination with other types of naomaterials are of considerable attention due to their novel properties [14]. Carbon nanotubes (CNTs), since discovered in 1991, have attracted considerable research interest in electrochemical biosensing fields due to their unique physical and electronic properties such as high electronic conductivity, high surface/volume ratio and unique ability to promote electron transfer $[15,16]$. CNTs and CNTs-based composites have been used to modify electrode for electrochemical sensing of hydrogen peroxide, glucose, dopamine, uric acid and ascorbic acid [17,18].

In this work, nanoflake-like $\mathrm{SnS}_{2}$ was synthesized according to previous method. Subsequently, a novel multiwall carbon nanotubes wrapped nanoflake-like $\mathrm{SnS}_{2}\left(\mathrm{MWCNTs}-\mathrm{SnS}_{2}\right)$ nanocomposite was proposed for the first time. The MWCNTs-SnS $\mathrm{S}_{2}$ composite modified electrode showed excellent electrocatalytic activity for hydrogen peroxide and oxygen. Moreover, the MWCNTs-SnS 2 composite promoted the direct electron transfer of glucose oxdase and the electrode surface.

\section{Experimental}

\section{Materials and Apparatus}

Tin (II) chloride dehydrate $\left(\mathrm{SnCl}_{2} \cdot 2 \mathrm{H}_{2} \mathrm{O}\right)$ and sulfur (S) were purchased from Sinopharm Chemical Reagent Co., Ltd. MWCNTs ( $\geq 98 \%$ purity, 60-100 nm diameter and 1-2 $\mu \mathrm{m}$ length) were bought from Shenzhen Nanoport Co. Ltd. GOD (EC 1.1.3.4, $108 \mathrm{U} \mathrm{mg}^{-1}$, from Aspergillus niger) was supplied by Amresco. D-(+)-Glucose and Nafion were purchased from Sigma-Aldrich.

Electrochemical measurements were performed on a CHI660B electrochemical workstation (Co., CHI, USA). Scanning electron micrographs (SEM) were obtained with a Hitachi S-4800 scanning 
electron microscope (Japan) at an acceleration voltage of $15 \mathrm{kV}$. Transmission electron micrographs (TEM) were obtained with a Philips Tecnai-12 transmission electron microscope (Holland) at an acceleration voltage of $100 \mathrm{kV}$.

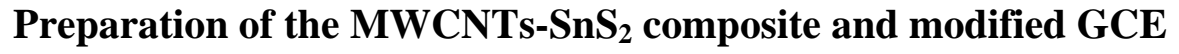

Nanoflake-like $\mathrm{SnS}_{2}$ was simply synthesized according to the previous method [19]. MWCNTs were firstly dispersed in $30 \%$ nitric acid and then refluxed for $24 \mathrm{~h}$ at $140{ }^{\circ} \mathrm{C}$. The resulting suspension was centrifuged, and the sediment was rinsed with water until the $\mathrm{pH}$ reached about 7.0 and dried in air at $60{ }^{\circ} \mathrm{C}$. Subsequently, $0.6 \mathrm{mg}$ of carboxylated MWCNTs and $0.2 \mathrm{mg}$ of yellow nanoflake-like $\mathrm{SnS}_{2}$ were dispersed together in $1.0 \mathrm{~mL}$ distilled water with ultrasonication for $0.5 \mathrm{~h}$ and then the suspension of $\mathrm{MWCNTs}^{-\mathrm{SnS}_{2}}$ was obtained. $5.0 \mu \mathrm{L}$ of the suspension of $\mathrm{MWCNTs} \mathrm{SnS}_{2}$ was dropped on the surface of the pretreated GCE and dried in silica gel desiccators. $5.0 \mu \mathrm{L}$ of $5 \mathrm{mg} \mathrm{mL}^{-1}$ GOD solution was then dropped on the MWCNTs-SnS 2 modified GCE and dried in silica gel desiccators. After $5.0 \mu \mathrm{L}$ of $0.5 \%$ Nafion solution was dropped on the GOD/MWCNTs-SnS 2 modified electrode surface, the GOD/MWCNTs-SnS $2 /$ Nafion modified electrode was finally obtained.

\section{Results and discussion}

\section{Characterizations of the MWCNTs-SnS ${ }_{2}$ composite}

The TEM and SEM images of the synthesized nanoflake-like $\mathrm{SnS}_{2}$ was shown in Fig. 1a and 1b, which exhibited an anisotropic and uniform flake-like morphology with about 40-nm flake

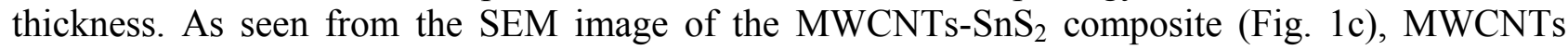
were well distributed on the surface of the nanoflake-like $\mathrm{SnS}_{2}$ so as to form the MWCNTs-SnS 2 nanocomposite, which was believed to be advantageous to enhance the electrocatalytic activity and electron transfer between the protein and electrode surface.

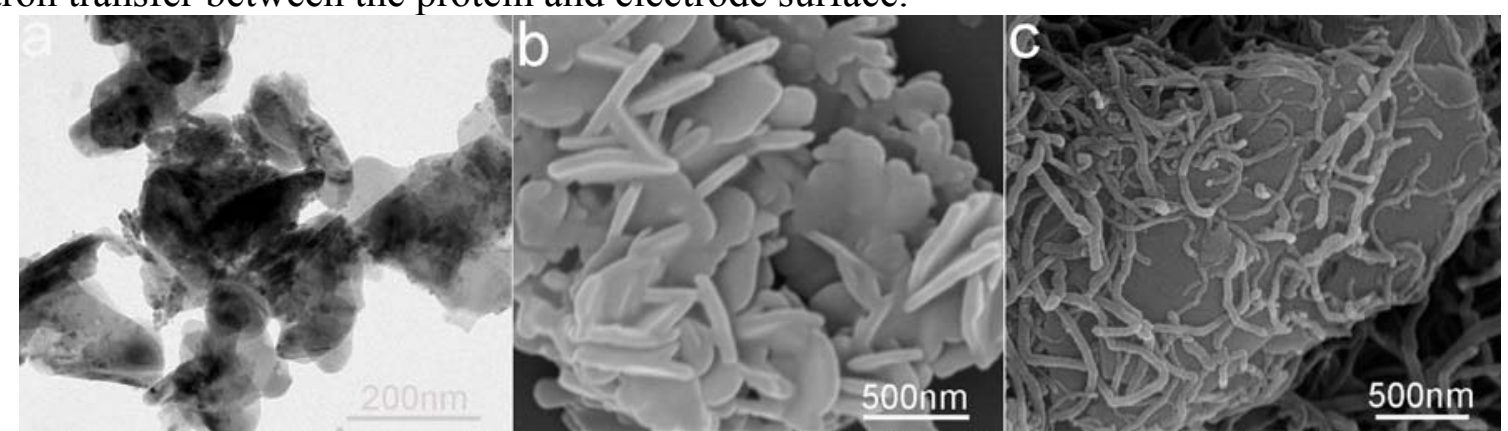

Fig. 1. TEM and SEM images of nanoflake-like $\mathrm{SnS}_{2}$ (a) and (b), and SEM image of

$\mathrm{MWCNTs} \mathrm{SnS}_{2}$ (c).

\section{The electrocatalytic behaviors at the MWCNTs-SnS 2 modified electrode}

Fig. 2 showed the cyclic voltammograms of different modified electrodes in $0.1 \mathrm{M}$ PBS. In the potential window from $-0.6 \mathrm{~V}$ to $+0.4 \mathrm{~V}$, no response was observed at GCE, while the MWCNTs-SnS 2 /GCE showed a pair of small redox peaks at -0.097 and $-0.083 \mathrm{~V}$, which is ascribed to the oxidation and reduction of the oxygen-containing group produced by the acid-treatment. Upon addition of hydrogen peroxide into the system, the GCE exhibited a weak response, while MWCNTs-SnS 2 modified GCE displayed a dramatically increasing reduction current.

Fig. 3 showed the cyclic voltammograms of different modified electrodes in $0.1 \mathrm{M}$ PBS. In $\mathrm{N}_{2}$-saturated 0.1 M PBS, no response appeared at the GCE, and the MWCNTs-SnS $2 /$ GCE showed a pair of characteristic redox peaks of the carboxylated MWCNTs. However, a current response was observed at GCE in air-saturated PBS, which began at $-0.3 \mathrm{~V}$ and reached the maximum at $-0.6 \mathrm{~V}$. Compared with the GCE, a more distinct reduction peak was observed at the $\mathrm{MWCNTs}^{-\mathrm{SnS}} \mathrm{S}_{2} / \mathrm{GCE}$ at $-0.51 \mathrm{~V}$, indicating an obviously enhanced electrocatalytic action for oxygen. 


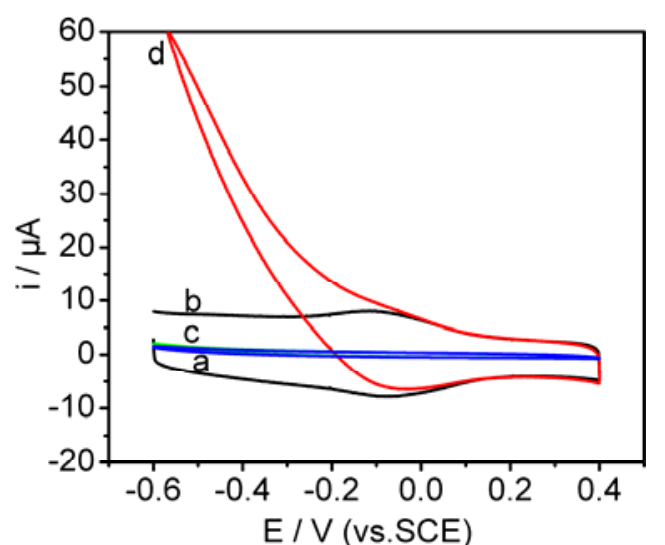

Fig. 2. Cyclic voltammograms of the GCE and MWCNTs-SnS $2 / \mathrm{GCE}$ at absence of hydrogen peroxide $(a, b)$ and in the presence of $5 \mathrm{mM}$ hydrogen peroxide (c, d) in PBS.

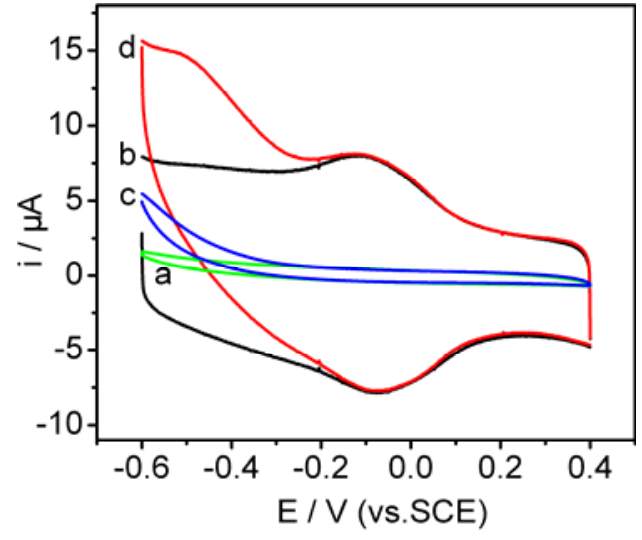

Fig. 3. Cyclic voltammograms of the GCE and MWCNTs-SnS $2 /$ GCE in $\mathrm{N}_{2}$-saturated PBS $(a, b)$ and in air-saturated PBS (c, d)

\section{Direct electron transfer of GOD at MWCNTs-SnS $\mathbf{~}_{\mathbf{2}}$ modified electrode}

In $\mathrm{N}_{2}$-saturated PBS, GOD/MWCNT-SnS 2 /Nafion/GCE displayed a pair of distinct and defined redox peaks at $-0.431 \mathrm{~V}$ and $-0.464 \mathrm{~V}$ (curve a in Fig. 4), suggesting the promotion of direct electrochemistry of GOD. In air-saturated PBS, the cyclic voltammogram of the GOD/MWCNTs-SnS 2 /Nafion/GCE showed a great increase in reduction peak current (curve b). When the glucose was added into this system, the reduction peak current of GOD decreased (curve c). Thus the glucose biosensor could be constructed based on the decease.

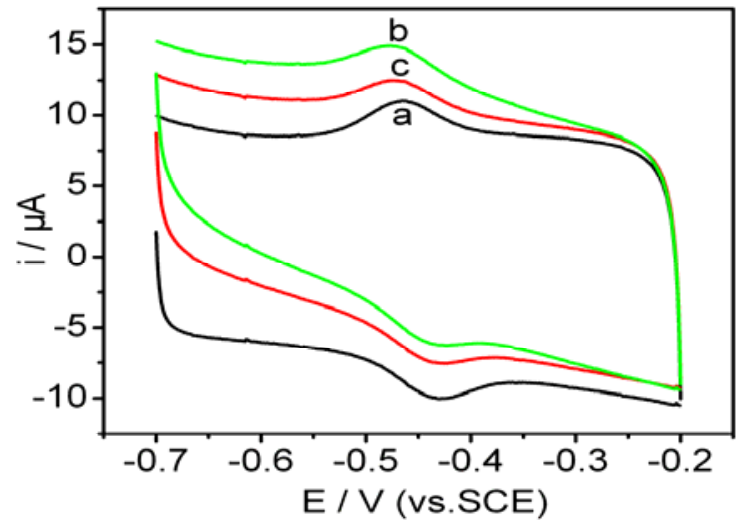

Fig. 4. Cyclic voltammograms of the GOD/MWCNTs-SnS 2 /Nafion/GCE in $0.1 \mathrm{M}$ pH 6.0 $\mathrm{N}_{2}$-saturated PBS (a), air-saturated PBS (b) and air-saturated PBS including $0.5 \mathrm{mM}$ glucose (c) at a scan rate of $50 \mathrm{mV} \mathrm{s}^{-1}$.

\section{Conclusions}

Here, a novel MWCNT-SnS $S_{2}$ nanocomposite was fabricated using a facile mixing approach. The

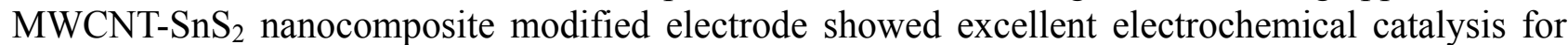
hydrogen peroxide and oxygen. When GOD was immobilized on the MWCNT-SnS $\mathrm{M}_{2}$ modified electrode, the direct electrode transfer could be achieved. The proposed MWCNT-SnS 2 composite provided promising alternative for electrochemical analysis and sensing.

\section{Acknowledgments}

This work was financially supported by from National Natural Science Foundation of China (21005070, 21075107), Postdoctoral Science Foundation of China (20110491462), Postdoctoral Science Foundation of Jiangsu Province (1101020B, 1102142C), The Priority Academic Program Development of Jiangsu Higher Education Institution, Open Research Fund of Jiangsu Key Laboratory of Environmental Material and Environmental Engineering (JHCG201009) and the Open Research Fund of State Key Laboratory of Bioelectronics of Southeast University (2011E13). 


\section{References}

[1] Q. Liu, X.B. Lu, J. Li, L. Yao, J.H. Li: Biosens. Bioelectron. Vol. 22 (2007), p. 3203

[2] H. Tang, F. Yan, Q.D. Tai, H.L.W. Chan: Biosens. Bioelectron. Vol. 25 (2010), p.1646

[3] G. Zhao, J.J. Xu, H.Y. Chen: Electrochem. Commun. Vol. 8 (2006), p. 148

[4] M. Yoshimoto, C. Lida, A. Kariya, N. Takaki, M. Nakayama: Electroanalysis Vol. 22 (2010), p. 653

[5] Z.H. Dai, G.J. Shao, J.M. Hong, J.C. Bao, J. Shen: Biosens. Bioelectron. Vol. 24 (2009), p. 1286

[6] A. Salimi, E. Sharifi, A. Hoorbakhsh, S. Soltanian: Biosens. Bioelectron. Vol. 22 (2007), p. 3146

[7] Z.J. Yang, X.C. Huang, Y.C. Zhang, J. Li, Q. Xu, X.Y. Hu: Electrochim Acta Vol. (2012) 70, p. 325

[8] N.G. Deshpande, A.A. Sagade, Y.G. Gudage, C.D. Lokhande: J. Alloy. Compd. Vol. 436 (2007), p. 421

[9] S.K. Panda, A. Antonakos, E. Liarokapis, S. Bhattacharya, S. Chaudhuri: Mater. Res. Bull. Vol. 42 (2007), p. 576

[10] C. Yang, W. Wang, Z. Shan, F. Huang: J. Solid State Chem. Vol. 182 (2009), p. 807

[11] T.J. Kim, C. Kim, D. Son, M. Choi, B. Park: J. Power Sources Vol. 167 (2007), p. 529

[12] J. Seo, J. Jang, S. Park, C. Kim, B. Park, J. Cheon: Adv. Mater. Vol. 20 (2008), p. 4269

[13] W. Shi, L. Huo, H. Wang, H. Zhang, J. Yang, P. Wei: Nanotechnology Vol. 17 (2006), p. 2918

[14] C.L. Sun, H.H. Lee, J.M. Yang, C.C. Wu: Biosens. Bioelectron. Vol. 22 (2011), p. 3146

[15] H.T. Zhao, H.X. Ju: Anal. Biochem. Vol. 350 (2006), p. 138

[16] X.C. Chu, B.H. Wu, C.H. Xiao, X.H. Zhang, J.H. Chen: Electrochim. Acta Vol. 55 (2010), p. 2848

[17] Z. Dursun, B. Gelmez: Electroanalysis Vol. 22 (2010), p. 1106

[18] H. Tang, J. Chen, S. Yao, L. Nie, G. Deng, Y. Kuang: Anal. Biochem. Vol. 331 (2004), p. 89

[19] Y.C. Zhang, Z.N. Du, S.Y. Li, M. Zhang: Appl. Catal. B: Environ. Vol. 95 (2010), p. 153 